\title{
Estudiar en inglés en universidades de países no angloparlantes: retos comunicativos y experiencias de la adaptación educativa y cultural en Lituania
}

\section{Studying in English at universities in non-English speaking countries: communicative challenges and experiences of educational and cultural adaptation in Lithuania}

Recibido: $12 / 01 / 2020$

Aceptado: 15/05/2020

Publicado: 26/06/2020
Beata Grebliauskienè

beata.grebliauskiene@gmail.com https://orcid.org/0000-0002-6504-4587

Universidad de Vilnius (Lituania)

Mariana Sueldo marsue@ism.1t

https://orcid.org/0000-0002-2078-0919

ISM University of Management and Economics (Lituania)

Resumen: El número de estudiantes que deciden estudiar en instituciones de enseñanza superior (IES) en el extranjero va creciendo de manera exponencial. Este crecimiento cuantitativo ha traído consigo una paulatina transformación cualitativa de la educación superior en una rama más de la industria global. Asimismo, crece la oferta académica de programas impartidos en lengua extranjera, mayormente en inglés. Si bien son cada vez son más numerosas las IES que procuran atraer nuevos alumnos con esta novedad, no todas están preparadas para afrontar debidamente el reto que supone la diversidad cultural del aula universitaria. Esta atractiva experiencia académica es, a la 
vez, un fenómeno rico en complejidad y cabe admitir que el impacto en los procesos de enseñanza y aprendizaje puede ser tanto positivo como negativo. El presente artículo se propone revelar los retos y problemas, de índole comunicativa e intercultural, a los que se enfrentan los estudiantes extranjeros que cursan estudios en inglés en universidades donde predomina el ambiente lingüístico y cultural lituano. Los datos empíricos obtenidos permiten sostener que difieren de manera considerable las experiencias y vivencias referidas a la causa y posibles soluciones a los retos mencionados, según se trate de la valoración de los alumnos extranjeros de los grupos focales o de lo que aportan las respuestas del personal entrevistado en la institución de acogida.

Palabras clave: Educación superior, Estudios en inglés, Estudiantes extranjeros, Adaptación cultural, Comunicación intercultural.

Abstract: The number of students who decide to enroll in Higher Education Institutions (HEIs) located away from their homeland is increasing every year across the globe. This quantitative change is bringing about a qualitative transformation as the HE sector is becoming one more globalized industry. Together with this growth, the academic offer of study programs delivered in a foreign language (most frequently-English) is also incrementing the level of complexity that HEIs while managing the cultural diversity of the university auditorium. HEIs are naturally lured into his attractive prospect to welcome a larger number of students; however, few are the HEIs truly ready and able to face the challenges that such complex phenomenon implies. A culturally diverse university class can certainly have both positive and negative impact on the teaching and learning processes. The purpose of this article is to explore how foreign students confront the communicational and intercultural challenges they encounter in a predominantly Lithuanian linguistic and cultural environment during their studies delivered in non-native English. The gathered and analyzed empirical data allow to state that there are considerable differences in the experiences and perceptions regarding the causes and possible solutions to the mentioned problems and challenges. The students who participated in the focus groups demonstrate contrasting viewpoints compared to those of the interviewed academic and administrative staff members in the receiving $H E I$.

Key words: Higher education, Studies in english, Foreign students, Cultural adaptation, Intercultural communication. 


\section{Introducción}

El creciente número de estudiantes que optan por estudiar en el extranjero está transformando la educación superior en una industria global. Se perciben marcadas tendencias de este constante crecimiento numérico (Cowley \& Hyams-Ssekasi, 2018). Ya en el 2004, aproximadamente 2 millones de jóvenes habían elegido cursar estudios superiores en el extranjero y para el año 2025, esta cifra podría llegar a los 8 millones (Altbach, 2004).

Con el fin de atraer un mayor número de estudiantes extranjeros, las instituciones de educación superior (IES) ofrecen programas de estudios en un idioma extranjero asequible a los futuros candidatos, quienes generalmente pueden manejarse, con cierta facilidad, en inglés. Por este motivo, en las últimas décadas, la lengua inglesa se ha convertido en la "lengua franca" global y predominante, la cual como afirma Graddol (2006), en muchos casos va perdiendo su estatus de lengua extranjera tradicional.

Es notable el crecimiento numérico de IES con ambiente cultural y lingüístico no angloparlantes que se suman a las filas de la oferta académica en inglés. Dado que, en estas instituciones, la mayoría de los estudios se cursan en el idioma local, la coexistencia con programas de estudios en inglés va paulatinamente transformando el ambiente interno en un espacio académico bilingüe.

En muchos países del mundo, las campañas de promoción para atraer estudiantes extranjeros a las universidades son ya una práctica habitual. No así en Lituania, donde todavía es un fenómeno más reciente. Según datos recabados por el Centro de Análisis y Seguimiento de la Educación Superior e Investigación de Lituania (MOSTA, 2016), el número de estudiantes extranjeros inscriptos en instituciones de educación superior (IES) de Lituania muestra un crecimiento de 300-400 estudiantes por año, siendo 6476 los estudiantes extranjeros cursando estudios en IES lituanas.

Una mayor proporción de alumnos internacionales trae consigo una mayor complejidad multicultural en las IES. Este fenómeno dejar ser algo excepcional tanto en el aula universitaria como para los profesores que trabajan con esta. Los estudiantes que conforman grupos académicos multiculturales cursan sus estudios en un idioma que no es su lengua materna, lo cual implica 
una necesidad de adaptación a sistemas diferentes de enseñanza-aprendizaje y de evaluación, a la vez que han de resolver los habituales problemas de una interacción condicionada por diferentes experiencias culturales. Los retos derivados de la diversidad cultural que estos estudiantes traen consigo (al incorporarse a las aulas en otro país y del impacto de la multiculturalidad en los procesos de aprendizaje y resultados académicos) han ido ganándose un cierto espacio entre los temas abordados por investigadores, cuya atención suele dirigirse especialmente hacia los problemas de adaptación a un nuevo ambiente cultural y académico.

Si bien no cabe duda de que las experiencias y retos con que se enfrentan los estudiantes extranjeros son objeto de estudio e interés para un número creciente de investigadores, se puede afirmar también que (en su gran mayoría) los trabajos de campo en este ámbito se llevan a cabo en universidades donde estudiantes locales y extranjeros comparten lengua y cultura, es decir, dentro de un ambiente monolítico. Empero, cada vez son más numerosas las universidades operantes en ambientes monolingüísticos y monoculturales, cuya oferta académica incluye programas de estudio impartidos en otro idioma (generalmente en inglés). Dicha oferta académica suele representar un porcentaje muy reducido de la totalidad de sus programas disponibles.

El ambiente lingüístico en estas universidades está marcado no ya por la lengua inglesa, sino por el idioma nacional del país, que en la gran mayoría de los casos es totalmente desconocida para los educandos extranjeros. Este es el caso de los estudiantes extranjeros que cursan estudios impartidos en inglés en las universidades lituanas. Cabe acotar que la comunidad lituano-parlante es relativamente pequeña: según datos del Departamento de Estadística de Lituania (2019), el país cuenta con una población de 2,794 millones de habitantes en 2019) y el uso de la lengua lituana se limita prácticamente a la geografía nacional de este país. Dado que los estudiantes extranjeros residen en Lituania durante su período de estudios (generalmente no más de un año y medio) la motivación para aprender el idioma local es muy baja. Esto implica un potencial reto tanto para los mismos estudiantes extranjeros como para las instituciones de acogida.

Estas últimas han de asumir la responsabilidad de crear las condiciones necesarias para una experiencia educativa y cultural exitosa y agradable a quienes forman parte de una minoría lingüística en un ambiente con predominio de 
la cultura e idioma lituanos. Por tanto, se puede inferir que los estudiantes extranjeros (que optan por estos programas) experimentarán algunos retos similares y otros únicos, en comparación con los que se enfrentarían si estudiaran en universidades de países angloparlantes.

En suma, el objetivo de este artículo consiste en revelar los retos y problemas de índole comunicativa e intercultural a los que se enfrentan los estudiantes extranjeros que cursan estudios en inglés en universidades donde predomina el ambiente lingüístico y cultural lituano.

\section{Diferencias culturales y su consecuente exclusión social}

Al explorar cuestiones relacionadas con la adaptación de los estudiantes, se ha extendido la práctica de estudios comparativos entre las vivencias de los estudiantes nativos/locales y los extranjeros. Cabe destacar que, si bien tanto unos como otros han de adaptarse a un nuevo ambiente y modos de interactuar, el desafío es indudablemente mayor para los no nativos, según los resultados de estudios realizados en universidades del Midwest de los Estados Unidos.

Se sostiene que los estudiantes extranjeros reciben menor apoyo social que los estudiantes locales (Hechanova-Alampay et al., 2002). Los alumnos venidos de otros países se encuentran lejos de su casa, familia y amigos; son relativamente pocos quienes logran formar un nuevo círculo de nuevos amigos y allegados en el país de acogida durante los estudios. La carencia de apoyo social dificulta su adaptación y esto, a su vez, puede tener consecuencias negativas respecto a los logros académicos.

Rajapaksa \& Dundes (2002) aportan resultados similares derivados del análisis de la adaptación de estudiantes extranjeros y estadounidenses matriculados en universidades de los Estados Unidos, enfatizándose la soledad y nostalgia hacia el país de origen y el hogar como los factores más relevantes de la experiencia narrada por los alumnos extranjeros. Por esta razón, Andrade (2006) afirma que los estudiantes extranjeros ponen sus expectativas en recibir una mayor o especial atención por parte de quienes los rodean: el personal administrativo y los profesores. 
Los estudiantes que cursan sus estudios en un país extranjero tienden a tener expectativas más altas también con respecto al desempeño de los profesores: alto nivel profesional en la asignatura que imparten y en sus dotes pedagógicas, y una especial consideración del profesor con los estudiantes extranjeros y sus necesidades particulares (Robotham, 1995). Lazos de amistad con gente del país de acogida naturalmente facilitarían la adaptación; sin embargo, lograr este tipo de relaciones resulta difícil.

Según datos empíricos, los alumnos que estudian en el extranjero tienden a crear tres tipos de redes sociales, cada una con una particular función de apoyo a su salud mental (Zhou et. al. 2008). Estas tres redes se denominan "monocultural", "bicultural" y "multicultural" y cobran gran relieve para una efectiva adaptación cultural (Furnham, 2004).

- La primera red se compone de estudiantes compatriotas y de amigos que dejaron en el país de origen, con quienes están vinculados por un bagaje cultural común que garantiza una comunicación fluida y un cierto nivel de bienestar psicológico.

- La segunda red (bicultural) se crea con las nuevas relaciones establecidas con personas nativas del país donde realizan sus estudios. Esta red es de suma importancia para comprender la cultura local y adaptarse a ella. Además, esto su vez influye, si bien indirectamente, en los logros académicos.

- La tercera red (multicultural) la conforman otros estudiantes extranjeros con experiencias culturales diversas y con quienes comparten el mismo reto ante un ambiente cultural nuevo que intentar conocer y comprender para lograr manejarse en él lo mejor posible.

Por el hecho de participar en los mismos (o relativamente similares) procesos de conocimiento y adaptación cultural, los miembros de esta red multicultural son de gran relevancia como fuente de información sobre la cultura de acogida, así como de posible apoyo social. La mencionada variedad de redes sociales facilita (en mayor o menor medida) la adaptación de los estudiantes extranjeros a la vida académica en el país de acogida, incluso cuando ellos mismos no hacen demasiados esfuerzos por establecer vínculos con los estudiantes locales y demás lugareños. Al-Shariden \& Goe (1998) sostienen que, en estos casos, los estudiantes extranjeros obtienen información sobre la cultural local mediante el trato con otros estudiantes que están en las mismas 
circunstancias y comparten cierta afinidad cultural o lingüística a través de la ya mencionada red multicultural.

La preferencia por las redes monoculturales o multiculturales puede atribuirse a la distancia cultural (Myles \& Cheng, 2003). Las numerosas diferencias culturales entre la cultura de origen y la de acogida influyen en el grado de dificultad para comunicarse con los nativos en el país de acogida. Por su parte, los grupos o comunidades con afinidad o experiencia cultural similar brindan confort y bienestar psicológico y emocional, reduciendo así la incertidumbre. Por tanto, ante una mayor similitud y afinidad, surge un sentimiento de comunidad que facilita la comunicación y permite centrarse en tareas de mayor trascendencia.

Así, la necesidad de socialización tiende a satisfacerse con la creación de vínculos en las redes monoculturales y multiculturales. Esta menor atención a la red bicultural puede ser un factor determinante de la exclusión social que separa a los estudiantes locales de los extranjeros. Estudios realizados en universidades de países angloparlantes demuestran que las diferencias culturales dificultan la comunicación entre locales y extranjeros. Una comunicación pobre y limitada trae como consecuencia la misma exclusión social y los dos tipos de estudiantes acaban cada uno por su lado, con escasa o nula interacción. De esto se puede inferir que los estudiantes extranjeros en universidades de países no angloparlantes se encontrarán con una situación analógica, de comunicación muy limitada con los locales y un cierto grado de exclusión.

\section{Nivel de competencia lingüística en inglés y la resultante exclusión co- municacional}

El idioma extranjero (inglés) demuestra ser un verdadero obstáculo para la puesta en marcha de programas de estudios impartidos en inglés (Halic et al., 2009). La competencia lingüística y las particularidades culturales afectan, sin duda, la adaptación al nuevo ambiente académico y los resultados en el proceso educativo. Los profesores que trabajan con alumnos extranjeros consideran que uno de los mayores desafíos es la competencia lingüística de estos alumnos (Trice, 2003). Muchos investigadores sostienen que la repercusión del conocimiento de la lengua va más allá de los logros académicos, abarcando también la adaptación social (Cowley \& Hyams-Ssekasi, 2018; Li et al., 2009; Andrade, 2006; Sawir, 2005; Robertson et al., 2000). 
Cursar estudios impartidos en inglés, cuando esta no es la lengua materna de los estudiantes, puede convertirse en un verdadero obstáculo que limita su participación activa en el proceso educativo y la comprensión del material de estudio. La competencia lingüística y su influencia en la adaptación y resultados académicos es un tema de alto interés para investigadores. Por esto, no es llamativo que se hayan realizado tantos estudios empíricos en las IES de Canadá, Australia, Estados Unidos y Gran Bretaña, donde estudiantes locales y extranjeros cursan sus estudios e interactúan en un mismo ámbito cultural y lingüístico.

Cabe señalar la unilateralidad de la cuestión sobre la competencia lingüística en la lengua inglesa en lo que respecta a la investigación sobre estudiantes extranjeros que cursan programas en ambientes culturales angloparlantes. El foco está en el dominio que los estudiantes extranjeros tienen del idioma, que es la lengua materna para la inmensa mayoría de los estudiantes locales, el personal académico y administrativo, así como de los habitantes del país en cuestión. Los angloparlantes, por tanto, no necesitan resolver barreras idiomáticas. Se puede afirmar que la competencia lingüística en inglés es uno de los posibles retos para los estudiantes extranjeros que cursan estudios en universidades de países no angloparlantes.

La competencia lingüística ha de ser analizada bajo diversos aspectos en lo que respecta a estudios impartidos en inglés en universidades de países no angloparlantes, puesto que (aun siendo la lengua franca) el inglés no deja de ser una lengua extranjera para casi todos los participantes en el proceso comunicativo. Entre los aspectos más determinantes destaca el nivel de inglés de:

- Los estudiantes: factor determinante de su capacidad para expresar su opinión y asimilar los contenidos del material de estudio.

- Los profesores: factor determinante de su capacidad para exponer los contenidos de la asignatura que imparten, para transmitir información adicional y mantener una comunicación fluido y continua con sus estudiantes.

- Personal administrativo: factor determinante de su capacidad para transmitir información necesaria y para resolver con solicitud los problemas que surjan. 
- Los demás estudiantes y los habitantes locales: ya que esto condiciona las posibilidades de interacción con los estudiantes extranjeros con el ambiente del país de acogida. En este caso la intensidad en el uso de la lengua extranjera es un punto clave condicionado por la (pre)disposición de los lugareños a comunicarse en inglés con los extranjeros.

A esto se añade el dominio de la lengua local por parte de los estudiantes extranjeros que les posibilita su participación en la vida social local. La falta de conocimiento básico del idioma local ya es de por sí una limitación que puede influir en la exclusión social, impidiendo o dificultando notablemente la comunicación activa con los lugareños y la participación en diversas actividades cuyo principal medio de comprensión es la lengua local. Lo mismo puede aplicarse a la competencia lingüística en inglés, solo que (en este caso) la posible exclusión social puede verse condicionada por el dominio de este idioma tanto de los estudiantes extranjeros como de los otros participantes en el proceso comunicativo.

El inglés suele ser la lengua franca y el único medio por el cual los estudiantes extranjeros pueden obtener la información necesaria, con más razón si la lengua local no es el inglés y los estudiantes llegan a otro país con un dominio muy pobre de este idioma. En este caso, cobra gran relevancia la accesibilidad a la información esencial en inglés. Obviamente esto no será un problema en un ambiente angloparlante nativo, donde toda la información estará disponible en inglés por ser el idioma local. Cuando el acceso a la información es limitado o insuficiente, puede surgir una exclusión informativa entre los estudiantes locales (quienes gozan de los recursos necesarios) y los estudiantes extranjeros que no saben el idioma local y, por tanto, resultan desfavorecidos por la carencia de recursos informativos.

\section{Métodos de enseñanza y aprendizaje, sus particularidades y retos}

Tal como demuestran estudios empíricos, la falta de familiaridad con los métodos de enseñanza y aprendizaje puede ser un factor problemático, desde el punto de vista de los profesores que trabajan con estudiantes extranjeros. Encuestas realizadas con estudiantes extranjeros en Australia y con sus profesores revelan que ambos agentes del proceso educativo difieren en su visión de los problemas que experimentan. Para los estudiantes, la razón de su 
escasa participación es la falta de conocimiento del inglés; por su parte, los profesores sostienen que la pasividad de los alumnos tiene su raíz en las particularidades culturales de los estudiantes, y no en el mayor o menor dominio del idioma (Robertson et al., 2000).

Asimismo, los profesores afirman que la experiencia previa del estudiante en su país de origen tiene un gran impacto en el proceso educativo, ya que los estudiantes extranjeros muestran preferencia por métodos de enseñanza y aprendizaje a los que ya estaban habituados (Andrade, 2006). Esta diferencia entre experiencias previas y nuevas en el aula (así como las preferencias de los estudiantes extranjeros por metodologías más afines) ha sido objeto de estudio de varios autores (Chan, 1999; De Vita, 2001; Robertson at al., 2000). Si bien cualquier método de enseñanza está (en mayor o menor grado) supeditado al contexto cultural donde se aplica (Chen \& Absalom, 1996) para los profesores que trabajan en aulas multiculturales, esto tiene consecuencias adicionales, ya que requiere mayor sensibilidad a las necesidades del educando y su bagaje sociocultural.

De gran ayuda puede resultar el conocimiento de las diferencias culturales entre el profesor y el estudiante para optar por la estrategia de enseñanza más idónea y sacar el máximo provecho de la sinergia cultural. Algunos subrayan que la participación activa de estudiantes occidentales en debates en el aula multicultural intimida a los estudiantes chinos (Pun, 1990). No obstante, Gilleard (1998) sostiene lo contrario, considerando el aspecto positivo de este método, aunque agrega que el mero debate por debatir sobre cuestiones que no añaden conocimiento alguno es visto como una pérdida de tiempo y, por tanto, desaconsejable. Ladd \& Ruby (1999) demuestran que una significativa mayoría de estudiantes (más de $80 \%$ ) enumeran la clase dictada por el profesor como el método principal de enseñanza, a la vez que expresan su preferencia por métodos más innovadores, como proyectos grupales, análisis de casos, entre otros.

Selvadurai (1992) ha explorado los problemas experimentados por los estudiantes extranjeros en las IES de Estados Unidos y subraya que la adaptación a un ambiente académico de diferente calidad (atmósfera distendida, mayor informalidad) implica un verdadero reto para los estudiantes extranjeros venidos de culturas cuyo proceso educativo es más formal y estructurado, donde la mayoría de los estudiantes simplemente escucha al profesor con mínima 
interacción. Gilleard (1998) afirma que los estudiantes asiáticos se enfrentan al reto de adaptarse a un sistema de enseñanza-aprendizaje más independiente y autónomo.

Algunos autores consideran que las distintas preferencias de estilos y métodos de aprendizaje guardan relación con características culturales tales como la distancia de poder. Una gran distancia de poder estimula a no pedir ayuda, no hacer preguntas y no contradecir incluso cuando no se comparten las ideas expuestas por el profesor. Por este motivo, los estudiantes chinos tienden a fiarse más del profesor y a su vez esperan recibir de él la información e instrucciones requeridas.

Asimismo, los alumnos chinos no son propensos a formular ni expresar sus propios puntos de vista, como suele suceder con estudiantes provenientes de culturas con una menor distancia de poder, como Gran Bretaña o Nueva Zelandia. Los estudiantes británicos y neozelandeses tienden a participar con mayor soltura e iniciativa en las tareas grupales y expresan su preferencia por los métodos de aprendizaje activos (Chan, 1999).

Las diferentes percepciones y expectativas con respecto a las relaciones profesor-alumno e interacción entre los alumnos se convierte en un problema recurrente para los estudiantes incorporados al proceso educativo en países extranjeros (Butcher \& McGrath, 2004). Engrosa la lista de los desafíos mencionados anteriormente el hecho de experimentar métodos de evaluación y valoración cuantificable que para algunos estudiantes extranjeros pueden resultar menos convencionales (De Vita, 2001). Según Gilleard (1998), las pruebas de control de asimilación de conocimientos (basadas en varias pruebas) también requieren una cierta adaptación y adquisición de habilidades nuevas y diferentes a las requeridas en exámenes basados en respuestas tipo ensayo.

Este último es quizás el método de evaluación al que están más habituados los estudiantes extranjeros. A raíz de una investigación empírica con estudiantes chinos de universidades australianas, Li et al. (2009) sostienen que dichos estudiantes se caracterizan por una participación menos activa en el aula; sin embargo, no han encontrado pruebas fehacientes para concluir que esta relativa pasividad sea la causa de resultados académicos insatisfactorios o inferiores. 
Los datos empíricos existentes no presentan conclusiones unánimes sobre la actitud u opinión de los estudiantes con respecto a nuevos métodos de enseñanza-aprendizaje y diferentes criterios de evaluación. No obstante, estos elementos del proceso educativo pueden llegar a ser un verdadero reto para los estudiantes que cursan estudios en universidades extranjeras. La novedad de los métodos educativos y los criterios de evaluación (anteriormente mencionados) no están necesariamente ligada al ambiente lingüístico, lo cual implica que también puede constituir un desafío para estudiantes que cursan programas en inglés en universidades no angloparlantes.

A modo de recapitulación, se puede afirmar la alta probabilidad de retos analógicos con los que se enfrentarán tanto los estudiantes que cursan programas en inglés en universidades no angloparlantes como los que estudian en países angloparlantes:

- Exclusión social: resultante de las diferencias culturales que dificultan la comunicación entre estudiantes extranjeros y locales.

- Insuficiente dominio del inglés: como obstáculo para el éxito en los estudios.

- Exclusión comunicacional: entre estudiantes extranjeros y locales debida al insuficiente dominio del inglés.

- Novedad de métodos educativos y criterios de evaluación: como obstáculo para resultados académicos más satisfactorios.

Cabe además prever otros retos particulares que afectarían especialmente a los estudiantes que cursan programas en inglés en universidades no angloparlantes:

- Exclusión comunicacional: suscitada por la competencia lingüística de profesores, personal administrativo y estudiantes locales, y por su disposición a usar el idioma inglés.

- Exclusión informativa de los estudiantes extranjeros: debido a la carencia de información disponible en inglés.

Con el fin de explorar las manifestaciones de los retos mencionados, se ha llevado a cabo una investigación empírica en una universidad de Lituania, la cual ofrece programas de estudios impartidos en inglés, junto a una amplísima oferta académica disponible en la lengua nacional del país. 


\section{Metodología de investigación}

El objetivo de la investigación es identificar y evaluar la importancia de los diferentes factores que afectan la adaptación educativa y cultural de los estudiantes que cursan estudios impartidos en una lengua distinta a la materna y fuera del país de origen.

El trabajo de campo pretende examinar las diferentes percepciones de los estudiantes con respecto a los estudios, métodos y estilos de enseñanza-aprendizaje que forman parte del sistema educativo del país de acogida y que, como tales, son un reflejo de la cultura local. Se pretende, asimismo, identificar los factores condicionantes de la adaptación educativa y cultural en el país elegido para cursar estudios universitarios. Se ha prestado especial atención a los siguientes aspectos:

- La asistencia ofrecida por las mismas universidades a los estudiantes extranjeros para facilitar su adaptación (tutores académicos, mentores, entre otros).

- El papel que juegan las competencias comunicativas de todos los agentes del proceso de enseñanza-aprendizaje (profesores, compañeros de curso, personal administrativo).

Para alcanzar el objetivo del trabajo empírico se ha recurrido a una combinación de métodos que permiten identificar la opinión y percepción de todos los participantes en el proceso educativo sobre los factores determinantes de la adaptación educativa y cultural. En concreto, se han realizado entrevistas cualitativas en profundidad y grupos focales. Estos últimos están integrados por 18 estudiantes extranjeros de variada procedencia, cursantes de programas de maestrías impartidas en inglés. Las entrevistas en profundidad se realizaron a 5 empleados administrativos y a 6 profesores que trabajan con estudiantes extranjeros con quienes se comunican en inglés.

Se ha escogido la entrevista en profundidad por ser un método que permite recabar material empírico más detallado sobre puntos de vista y experiencias de los informantes. En ese sentido, se elaboraron las siguientes preguntas:

- Q1: problemas relacionados con la competencia lingüística y predominio de la lengua lituana. 
- Q2: diferencias culturales como obstáculos de la comunicación.

- Q3: tipo de cuestiones por las que los estudiantes se dirigen al personal administrativo de la universidad.

- Q4: trato entre los estudiantes locales y extranjeros; cómo fomentarlo.

Se añadió una pregunta a la lista destinada a los profesores:

- Q5: métodos de enseñanza en ambiente multicultural y retos para el profesor.

El método de discusión en grupo focal suele considerarse como un tipo de entrevista que permite abarcar y comprender (en mayor profundidad) las experiencias individuales de los participantes y, con su ayuda, reconstruir situaciones concretas (Flora Hung, 2004). Según Morgan (1997), este método también es recomendable para sondear nuevas ideas, opiniones, sentimientos y emociones de diversas personas sobre fenómenos o procesos. Esto facilita llegar a los factores que influyen en dichas opiniones, comportamientos y motivaciones.

Cabe acotar que una de las principales ventajas del grupo focal es precisamente la dinámica grupal donde se da una interacción e intercambio de conocimientos y vivencias que contribuyen a generar nuevas ideas. De este modo, se supera la mera recolección de datos sobre las experiencias individuales de los participantes para dar lugar a una profundización, complementación y explicación mutua sobre las emergentes diferencias de opiniones y experiencias. Las particularidades de este método son muy relevantes a la hora de investigar la interacción social (Kleiber, 2004).

Para los grupos focales, se ha utilizado un cuestionario destinado a identificar las experiencias de los estudiantes sobre los siguientes aspectos:

- Influencia de la competencia lingüística en inglés sobre el proceso de aprendizaje y la comunicación.

- Problemas y ventajas del uso del inglés en el proceso de aprendizaje.

- Aspectos positivos y negativos de la alta densidad de diversidad cultural en el grupo académico.

- Buenas prácticas y problemas relacionados con la aplicación de métodos de enseñanza y aprendizaje. 
- Integración en la comunidad académica local y la influencia de las diferencias culturales sobre dicha integración.

- Accesibilidad de información en inglés y satisfacción de la necesidad de información.

- Predominio de la lengua lituana y los retos que esto supone.

Los datos obtenidos, a través de las entrevistas cualitativas (grupales e individuales), han sido analizados aplicando el método de análisis de contenidos con el objetivo de identificar los factores más esenciales que condicionan el rol de los estudiantes como agentes principales del proceso de enseñanza-aprendizaje y su interacción con los demás agentes de este proceso. El análisis cualitativo de contenido textual hace posible la identificación de temas esenciales y posibles correlaciones entre estos, así como de los roles de los agentes y el contexto en que tiene lugar la interacción. Las aportaciones de los informantes se presentarán en letra cursiva e indicando con letras y números al final de la cita según se trate de "estudiantes" $(\mathrm{E})$, "profesores" $(\mathrm{P})$ o "personal administrativo" (A).

Durante la investigación empírica, se han detectado ciertas limitaciones atribuibles al distinto nivel de inglés de los informantes, a sus experiencias educativas anteriores en los países de origen, sus diversas competencias comunicativas y características personales. En el marco de la presente investigación, no se examina ni se evalúa el ambiente cultural particular del que proviene cada uno de los entrevistados, aunque naturalmente esto puede haber tenido cierta influencia en los resultados obtenidos.

\section{Resultados de la investigación empírica y discusión científica}

\subsection{Retos derivados de la competencia lingüística en inglés}

A diferencia de los estudiantes que cursan estudios en universidades de países angloparlantes, los entrevistados que estudian programas impartidos en inglés en universidades no angloparlantes no mencionan la competencia lingüística como uno de sus retos. Afirman que, para sus profesores, el inglés tampoco es su lengua materna y, por tanto, los docentes se solidarizan más con la situación de los estudiantes pues comprenden que este idioma extranjero es la lengua común que les permite trabajar juntos. 
Así, el hecho de que el inglés no sea la lengua materna de los profesores no constituye obstáculo alguno para los alumnos. Es más, consideran que el conocimiento de las materias y la competencia lingüística de sus profesores son de alto nivel. Algunos mencionan que al elegir estudios impartidos en inglés tenían cierto temor respecto a posibles dificultades de comprensión o malentendidos:

Antes tenía un poco miedo. Había oído que algunos profesores dan su clase simplemente leyendo apuntes ... O que tenían un acento tan marcado al hablar inglés que era muy difícil entenderlos ... Pero me llevé la grata sorpresa con su alto nivel de inglés y en general buen nivel de conocimiento. Realmente lo disfruto. (E1)

Sin embargo, cabe señalar que hablar en un idioma extranjero con acento no nativo (lo cual es frecuentemente inevitable) sí dificulta la comunicación en un ambiente multicultural. No obstante, sería infundado concluir que esta dificultad es aplicable a todos indistintamente. Mientras para algunas culturas el hecho de que los extranjeros tengan una pronunciación menos nativa cuando hablan en otro idioma puede facilitar la comprensión, para otros puede ser un obstáculo:

A veces me resulta difícil seguir lo que están diciendo porque hablan con un acento muy fuerte y me cansa. (E10)

En cambio, a mí, por ejemplo, el acento de los profesores me resulta comprensible, porque se parece al nuestro ... aunque a los estudiantes de otros países les resulta molesto. (E12)

A mí me es más fácil entender el acento local que el de los angloparlantes nativos. (E17)

La variedad de pronunciación de cada profesor también puede ser un reto por tener que adaptar el oído a acentos particulares:

Casi todos hablan con un acento diferente y, a veces, es realmente un desafío entenderlos. (E15)

Los estudiantes generalmente resuelven este problema comunicacional por sus propios medios, procurando escuchar con más atención, buscando material de lectura, entre otros aspectos: 
Me parece poco educado pedirle al profesor que repita cuando no lo entiendo. (E15)

El nivel de inglés de los mismos estudiantes es, a veces, un obstáculo para sus estudios. Debido a su insuficiente competencia lingüística, algunos carecen de confianza en sí mismos. Por ese motivo, piden ayuda a los compañeros de aula:

Tenemos un grupo de amigos de Italia a quienes les cuesta bastante entender lo que explica el profesor, así que se sientan al lado de otro que entiende y les traduce todo. (E10)

Los profesores también consideran que uno de los mayores obstáculos es la competencia lingüística en inglés. El insuficiente dominio de esta lengua impide a los estudiantes formular y expresar con fluidez sus ideas y participar activamente en los debates u otras tareas:

El bajo nivel de inglés de unos dificulta la discusión con los demás estudiantes y se convierte en impedimento para asimilar conocimientos. (P3)

La capacidad para expresarse en inglés es muy dispar. (P1)

Esta disparidad, aunque no necesariamente relacionada con la lengua materna de los estudiantes, constituye un desafío para los profesores, ya que

todos tienen que aprender y el profesor ha de resolver la cuestión y decidir dónde enfocar sus esfuerzos y atención para que nadie se sienta dejado de lado ... la diferencia de nivel en el uso del idioma acaba también afectando su propia satisfacción. (P3)

Los profesores tienden a asociar la insuficiente competencia lingüística en inglés con la necesidad de hacer mayores esfuerzos para exponer y explicar los contenidos y mantener la discusión o los debates, aunque esto no siempre afecte los logros académicos de los estudiantes.

Cabe destacar que estas respuestas difieren notablemente de las aportaciones de autores que examinan los problemas generados por los distintos niveles de competencia lingüística de los estudiantes extranjeros en universidades 
angloparlantes. Los estudiantes extranjeros en universidades no angloparlantes no suelen considerar que su dominio del idioma sea un asunto de gran entidad. Al ver que el inglés es la lengua franca y (salvo contadísimas excepciones) no es la lengua materna del entorno, los estudiantes extranjeros no suelen preocuparse demasiado por su propia pronunciación, falta de fluidez o limitado vocabulario. El inglés tampoco es la lengua materna de sus profesores, quienes tienden a ser menos exigentes y brindarles más ayuda.

El uso de la lengua inglesa (como medio de comunicación) conlleva una cierta exclusión que suele sorprendentemente manifestarse también en la relación profesor-alumno, particularmente en la dimensión del apoyo brindado al estudiante:

Sobre la supervisión de tesinas de maestría. Los que estudian en lituano y son responsables reciben mucha más ayuda y material más detallado que los que cursan en inglés. (E10)

Además, me enteré que si te diriges al profesor en persona, te ayuda mucho más que si se lo pides por escrito. (E12)

Cabe señalar que estos problemas guardan más relación con la abundante información solicitada por los estudiantes extranjeros que con el idioma.

\subsection{Métodos de enseñanza y aprendizaje}

Los métodos educativos tampoco aparecen en la lista de retos enumerados por los alumnos, lo cual puede resultar sorprendente si se tiene en cuenta que en la discusión grupal participaron representantes de culturas individualistas (con baja distancia del poder) y otros de culturas colectivistas, con alta distancia del poder. Conocer nuevos métodos de enseñanza y aprendizaje forma parte de las expectativas de estos estudiantes para quienes el cambio de métodos no supuso dificultad alguna:

Estábamos preparados para algo nuevo, era lo que esperábamos. (E2)

Los estudiantes asocian los cambios de métodos de enseñanza y estudio con el cambio de ciclo de estudios: 
Hay una diferencia esencial entre los estudios de grado y las maestrías. (E4)

Me gusta la orientación práctica ... Tuvimos estudios de casos reales, de problemas reales. (E2)

Todos los estudiantes extranjeros afirman tener estilos concretos de aprendizaje y sostienen que algunos nos les resultan muy efectivos. Los métodos de evaluación y de enseñanza-aprendizaje que son útiles y efectivos para los estudiantes locales no necesariamente lo serán para los extranjeros (Cowley \& Hyams-Ssekasi, 2018). Sin embargo, los estudiantes consideran que el profesor no está obligado a tener en cuenta el estilo individual de cada alumno, pero a su vez afirman que

si bien el profesor no tiene que adaptar sus métodos a los estudiantes, al menos tendría que explicar con suma claridad lo que espera de los estudiantes. (E15)

Los profesores también subrayan que la causa del problema no yace en los métodos de enseñanza y aprendizaje en sí mismos, sino más bien en la gran diferencia de comprensión cuando se explica cómo llevar a cabo tareas y actividades de la clase, pues esto presupone experiencias previas como punto de referencia y comparación para entenderlas. Los estudiantes locales cuentan con el bagaje de su experiencia en la escuela secundaria o estudios de grado. Por tanto, se sienten más seguros, mientras que los extranjeros necesitan explicaciones más detalladas:

Hay una gran diferencia de experiencias. Los estudiantes locales enseguida entienden lo que tienen que hacer cuando se les asigna una tarea. En cambio, los extranjeros necesitan más tiempo para orientarse ... hay que darles instrucciones más detalladas. (P3)

A veces los extranjeros requieren información exhaustiva sobre cuestiones elementales y evidentes para los locales:

En nuestro sistema educativo no existen los trabajos finales, tipo tesinas. En cambio, aquí tienes que escribir la tesis de máster y no sé cómo hacerlo. Sé que debo hacerlo por mi cuenta, ser más autónomo, pero ¿cómo? Para mí esto es realmente un desafío. (E13) 
Hay un gran desfase y un vacío de conocimiento sobre cómo escribir los trabajos de fin de curso o tesinas ... Y los modelos de trabajos ya escritos por otros de poco me sirven. Te enteras de cómo tiene que ser, pero ¿cómo elaborar uno tú mismo? ... Y las búsquedas en Internet tampoco ayudan, porque encuentras miles de formas diferentes y no te queda claro qué te piden aquí. (E10)

Esta diversidad de bagaje entre estudiantes locales y extranjeros puede motivar la necesidad que estos últimos tienen de comunicación más frecuente para solicitar apoyo e información más detallada. Durante la discusión con los grupos focales se hizo hincapié en las orientaciones que se dan en el aula durante el proceso educativo. Los estudiantes extranjeros sostienen que en un ambiente de alta complejidad cultural es de gran utilidad contar con orientaciones claras y válidas para todos. Este tipo de orientaciones deben ser inequívocas y comprensibles para los representantes de diferentes culturas, sin dar espacio a distintas interpretaciones.

Los estudiantes extranjeros expresan cierta insatisfacción ante la ausencia de este tipo de instrucciones y orientaciones, pues les impiden realizar bien sus tareas, lo cual luego repercute negativamente en la evaluación de sus trabajos. Debido a esta debilidad, muchos entienden la tarea asignada de una manera totalmente distinta y la llevan a cabo según su entender. Esto realza la importancia del rol del profesor del aula multicultural como principal y eminente fuente de información, ya que los estudiantes no cuentan con sus pares locales.

Los estudiantes también trataron sobre el feedback que les habría gustado recibir junto a comentarios constructivos sobre sus trabajos realizados. Hallazgos empíricos en universidades de países angloparlantes ilustran (con casos concretos) la marcada necesidad de los estudiantes llegados al país extranjero de recibir explicaciones exhaustivas, comentarios y orientaciones detalladas para poder realizar las tareas asignadas. Según Andrade (2006), los estudiantes sostienen que los comentarios de sus profesores (sobre trabajos presentados para evaluación) son uno de los métodos educativos más útiles y recomendables:

Estos estudiantes requieren mayor atención, tienes que dedicarles más tiempo y trato. (A1) 
Se puede afirmar que los métodos, per se, no son un problema, pues los estudiantes se muestran abiertos y expectantes ante las novedades educativas del nuevo ambiente. El éxito en la realización de los trabajos y tareas que se les asignan depende de cómo y con cuanta claridad se les explican las consignas para que puedan llevarlos a cabo. Esto requiere una alta dedicación por parte del profesor, quien ha de comunicarse con los alumnos con mayor asiduidad e intensidad durante todo el período de estudio, a fin de discernir lo que los estudiantes no saben o no entienden bien.

\subsection{Alta densidad de diversidad cultural como reto comunicativo}

Durante la discusión sobre sus experiencias y problemas, los estudiantes no hicieron mención de la adaptación cultural como una dificultad, aunque sí consideran un reto tener que adaptarse a las diferencias y diversidad culturales. Pero este desafío es de una índole distinta al que experimentan los alumnos extranjeros que cursan estudios en una cultura angloparlante. En dicho ambiente académico, los estudiantes comparten el proceso educativo con una considerable proporción de estudiantes locales y un menor número de otros países.

En contraste, los alumnos que cursan programas impartidos en inglés en universidades de países no angloparlantes se encuentran con una alta densidad de diversidad cultural dentro de su grupo académico, al que se incorporan con escasa experiencia previa de comunicación intercultural e incipientes aptitudes de trabajo en grupos multiculturales. Estos alumnos han de aprender a arreglárselas por sí mismos para superar los obstáculos de comunicación intercultural a través de la interacción en su grupo académico.

El uso de métodos activos de enseñanza pone en mayor evidencia la importancia de las aptitudes y de la falta de experiencia previa antes mencionadas. En grupos de trabajo multiculturales, los estudiantes han de realizar las tareas asignadas y gestionar simultáneamente sus dificultades de comunicación intercultural, contando con poca experiencia y ninguna ayuda externa al grupo:

Al principio, no me gustaban los proyectos grupales porque no sabía trabajar en grupo ... pero después de un tiempo realmente me empezó a gustar y mucho. (E6) 
Los métodos activos de enseñanza dejan de crear tensión una vez superados los problemas comunicativos que surgen de las diferencias culturales y habiendo alcanzado un mejor conocimiento mutuo:

Aprendimos mucho durante todos esos proyectos. Cómo trabajar con gente tan distinta, cómo gestionarlo. (E1)

Los métodos activos de enseñanza (junto con las actividades extracurriculares) brindan la oportunidad de un trato más asiduo entre los mismos estudiantes, además de ser ocasión para conocerse mejor e intercambiar información cultural:

Cuando vine aquí por primera vez vi mucha gente distinta. Sí, somos compañeros de curso, pero ... después de una velada cultural nos hicimos más amigos. (E12)

Por la particularidad de los estudios, se dedica mucho tiempo al estudio individual y se fomenta menos la socialización espontánea, incluso dentro del mismo grupo académico:

Te encuentras con el grupo con poca frecuencia, pocas veces por semana ... pues, llegamos, estamos en la clase y luego cada uno a lo suyo. (E10)

Sin un incentivo externo para comunicarse entre sí, los estudiantes tienden a reunirse en grupos más reducidos con quienes se sienten más cómodos, generalmente por una cierta afinidad cultural. En todo caso, falta trato:

Si alguno necesita algo relacionado con el material de clases, se ponen en contacto contigo, te buscan. Pero para otros asuntos... no les interesa. (E13) Me siento parte de un grupo de extranjeros, más que de un grupo de compañeros de curso. Ahora que lo pienso, no tenemos ni siquiera un grupo de Facebook. (E17)

Los grupos focales han permitido comprobar que, si bien es un factor importante, la mera pertenencia a un mismo grupo académico (con la consiguiente participación en las clases) no implica que se den las condiciones necesarias para una comunicación asidua entre los estudiantes. Así, el uso de métodos activos de enseñanza fomenta una mayor intensidad de interac- 
ción, pero también hacen más evidente la falta de competencia y conocimiento interculturales.

Por otro lado, los profesores se percatan de la mayor flexibilidad y comprensión cultural requerida por sus estudiantes:

Es una cuestión cultural, porque los estudiantes vienen con ciertas expectativas y esperan que se tengan en cuenta sus particularidades. (P2)

Cuando surgen problemas comunicacionales (derivados de las diferencias culturales, especialmente en los trabajos grupales y tareas que requieren mayor interacción), los estudiantes tienden a dirigirse al profesor y esperan su ayuda para resolverlos:

Yo misma tengo que tomar conciencia sobre el tipo de alumnos con quienes estoy trabajando, cuáles son sus características, sus diferencias, cómo me entiendo con ellos, y cómo logran ellos conectar conmigo y a la vez velar también para que ellos logren interactuar entre sí ... y ayudarles en lo que pueda. (P3)

En un grupo de alta densidad multicultural, el profesor asume el rol de consultante o facilitador de comunicación intercultural, con el fin de garantizar que el proceso de enseñanza-aprendizaje llegue a buen término. Esto implica que el profesor ha de contar con conocimientos especializados y alta competencia intercultural.

\subsection{Exclusión social condicionada por la cultura}

Una de las vivencias negativas mencionadas por los estudiantes es la exclusión. No se sienten parte de la vida universitaria:

Hay una participación del 0\% en la vida universitaria. (E9)

Yo solo vengo a clase. (E2)

A decir verdad, me siento más miembro de la comunidad de ESN [Erasmus Student Network] que de la comunidad universitaria ... Porque la ESN nos incluye en todo tipo de eventos con más frecuencia que la universidad. (E12) 
Me siento parte de la universidad, pero, en realidad, cómo te lo digo ... parece que la universidad tiene una línea para los que estudian en lituano y otra para los que estudian en inglés ... y estas dos líneas no se intersecan. (E12)

El desarrollo de programas de estudios impartidos en inglés crea un problema imprevisto que no se resuelve con la mera participación en programas de intercambio estudiantil. Los estudiantes inscriptos en programas de intercambio suelen permanecer en la universidad asociada que los acoge un semestre o como mucho dos. Más allá de sus actividades académicas, estos invitados temporales demuestran cierto interés por la cultura local. En cambio, los estudiantes extranjeros "permanentes" necesitan sentirse parte de la comunidad universitaria al igual que los demás estudiantes locales, lo cual resulta bastante difícil de lograr:

Sí, hay algunas opciones si quieres participar ... pero todo es en puro lituano ... Yo quería asistir a uno de los clubes, pero cuando fui, todo era en lituano y yo era la única extranjera. Solo te queda una opción: el club de alumnos extranjeros. Pero no quiero socializarme solo con extranjeros. Me interesa conocer a lituanos. Estoy viviendo aquí y quiero establecer lazos con los de aquí. Si alguna vez necesito algo, me pueden ayudar, pero... (E9)

La inclusión de los alumnos extranjeros en la vida universitaria común a todos los estudiantes es un problema condicionado por el idioma. Los extranjeros cursan sus estudios en inglés y no saben la lengua utilizada por los alumnos locales y en la mayoría de programas de la oferta académica. Los alumnos locales hablan inglés muy bien, pero tienen su propio ritmo de vida (y en su idioma local), por lo cual los extranjeros tienen muy poco trato con ellos.

La exclusión se manifiesta no solamente en la organización de actividades extracurriculares por separado para los respectivos grupos, sino también en el desarrollo de los estudios:

Es muy común que los profesores tengan clases con los lituanos por la mañana y las mismas clases con los extranjeros en inglés por la tarde. (E12) 
Esta exclusión surge de manera natural, debido al idioma en el cual se imparten las clases. Ciertamente, hay alumnos locales en los programas impartidos en inglés, pero no dejan de ser una gran minoría.

La interacción entre estudiantes locales (que estudian en lituano) y los extranjeros (que lo hacen en inglés) se fomenta a través de asignaturas electivas o sesiones especializadas impartidas en inglés. Sin embargo, a los estudiantes locales no les apetece demasiado participar. (A1)

Ante la carencia de contacto con estudiantes locales, la necesidad de apoyo social se traslada al personal académico y administrativo, que representan la cultural local y suelen ser conocidos por los estudiantes extranjeros y los hacen sentirse seguros:

A veces pasa que los estudiantes vienen a nuestro despacho o al de algún profesor con el solo fin de pasar un rato y tener con quien charlar. (A1)

A los alumnos interesados en participar más activamente en la vida universitaria se les brindan algunas oportunidades, pero todas se organizan por separado de las actividades para los locales. Es natural que los estudiantes locales (que hablan lituano) tengan sus actividades extracurriculares en su propio idioma. Los que estudian en inglés son un porcentaje muy reducido respecto del total de estudiantes. De este modo, coexisten dos vidas paralelas que prácticamente nunca convergen, por lo cual no se dan ocasiones de interacción entre extranjeros y nacionales.

Esta falta de comunicación, a su vez, aumenta la dificultad de entablar nuevos lazos y de expandir la red social, fomentando indirectamente la polarización de los estudiantes extranjeros que tienden a relacionarse sólo entre ellos y a no interactuar con los locales. Esto da pie a que se expanda la red multicultural en detrimento de la bicultural, lo cual a su vez hace más difícil la efectiva adaptación cultural de los estudiantes extranjeros.

Otro factor que agudiza la exclusión son las residencias o alojamientos estudiantiles también separadas para estudiantes locales y extranjeros respectivamente, por lo que la vida estudiantil diaria también transcurre por separado: 
Hay dos tipos de residencias universitarias: unas para lituanos, otras para extranjeros. En nuestro alojamiento no hay lituanos. (E12)

Esta separación física tampoco crea condiciones para una interacción natural y espontánea con el modo de vida local. En opinión de los estudiantes, esta separación tiene su aspecto negativo, pues a todos los foráneos se los reúne en un mismo grupo, etiquetados indistintamente como "extranjeros", independientemente de que unos sean estudiantes de intercambio temporal por un semestre o que hayan venido al país para hacer toda la carrera en la universidad.

Si bien es cierto que no dominar la lengua local supone muchos inconvenientes, los estudiantes extranjeros no muestran mucho afán por aprender el idioma. Se les ofrecen cursos de lituano, pero son muy pocos los que aprovechan esta oportunidad.

Son muy pocos los que se interesan por esto ... tal vez un $2 \%$ de todos los estudiantes. (A5)

\subsection{Exclusión informativa debido al idioma}

Asimismo, se identifica una exclusión informativa de los estudiantes que cursan programas en inglés, considerado como lengua franca. Esto se percibe principalmente en las indicaciones y señalización del espacio físico donde tiene lugar la actividad académica:

Lo que veo y no deja de sorprenderme es la falta de uso del inglés en la universidad. En general, todas las salas, clases, oficinas, todos los cargos y puestos del personal están indicados en lituano. (E1)

Esta falta de información supone un serio obstáculo para encontrar al personal especializado o acudir a las áreas concretas o incluso para encontrar el aula donde tienen clases:

[El coordinador] nos avisó que el martes tendríamos clases en el aula "La Voz de Vilnius" ... pero no había indicaciones claras para encontrar la dirección. ¿Y yo cómo sé dónde está esa aula? (E6) 
Se constata la falta de señalización en inglés en las instalaciones, por más que la universidad pretenda ser un espacio bilingüe. Este inesperado inconveniente se agrava a la hora de identificar las aulas que llevan nombres o denominaciones en lituano:

Los nombres de las aulas están todos en lituano. (A1)

Casi todas las aulas tienen nomenclaturas numéricas incomprensibles para los extranjeros. Sin embargo, el problema surge no sólo con los nombres de las aulas, sino también con los carteles de las oficinas del personal administrativo. También es un verdadero reto para los estudiantes extranjeros comprender las instrucciones que reciben en lituano (la lengua nacional) y no en inglés. Naturalmente, la información dirigida al alumnado está en el idioma oficial del país, pero no todos los estudiantes la entienden:

Si quieres ir a la cafetería, allí todo está escrito en lituano ... el problema surgió ya al comienzo de los estudios. Por eso pusieron el menú en inglés, pero en realidad allí no hay nada... (E1)

En la biblioteca encontré instrucciones para el uso de equipos (impresoras, fotocopiadoras, lo que sea) pero todo en lituano ... Dice: "Introduzca su nombre de usuario, contraseña, número de carnet de identidad" ... ¡Todo en lituano! Si uno no está acostumbrado a estos sistemas, no sabe qué poner... (E5)

Este tipo de problemas se soluciona en parte gracias al personal cualificado de la universidad que se maneja perfectamente en inglés y está disponible para consultas y ayudar en lo que haga falta:

En la mesa de entrada de información todos hablan inglés y te pueden explicar y ayudar. Pero, ¿por qué tengo que pedir ayuda? (E7)

Esto me incomoda, me siento confusa. ¿Por qué he de buscar alguien que me ayude cuando podría arreglármelas por mi cuenta si tuviera la información adecuada y disponible? (E5)

Para algunos estudiantes, el hecho de requerir ayuda es una experiencia desagradable o al menos fuera de su zona de confort, mientras que para otros no les supone ningún inconveniente. En cualquier caso, la falta de información conlleva una cierta limitación de autonomía y el sentimiento de dependencia: 
Y entonces te haces dependiente de los demás. (E2)

Otro obstáculo de índole comunicacional es la falta de información sobre el uso de programas informáticos, plataformas digitales y bases de datos. Toda la información requerida para el correcto uso de estos medios está disponible solamente en lituano, puesto que la gran mayoría de los usuarios saben este idioma, mientras que los angloparlantes son un porcentaje muy reducido:

Una cuestión problemática es la de las plataformas digitales que necesitamos para los estudios. Ya que mayoría de los estudiantes extranjeros tienen que estar permanentemente preguntándole a los lituanos cómo usarlas y todo eso. Debido a la real falta de información, los estudiantes preguntan todo el tiempo como encontrar esto o aquello, como registrarse, o acceder a tal sitio. (E1)

Surgen otros problemas a la hora de navegar por páginas de Internet, pues las diferencias socioculturales traen consigo distinto grado de alfabetización informática. Lo que parece fácil e intuitivamente comprensible para los locales, puede resultar un verdadero impedimento para los demás:

Al principio cuesta bastante aprender a usar el horario electrónico. (E6)

Asimismo, no toda la información relevante está disponible en inglés:

La información sobre becas está solo en lituano ... puedes abrir la página e intentar con traductor de Google ... y lo mismo con la información sobre prácticas ... todo en lituano, o sea sólo disponible en la versión lituana del portal. (E12)

Hay muchísima información sobre eventos, incluso muchos multiculturales, pero todo solamente en lituano. (S10)

¿Cómo me enteré de las fechas tope y la documentación requerida para el programa de intercambio ERASMUS? ... Descubrí un cartel en el primer piso donde logré detectar una palabra más o menos comprensible por su parecido con mi propio idioma. Entonces fui al despacho de la secretaria y conseguí los datos que necesitaba ... pero otros estudiantes no tuvieron esta posibilidad. (E12) 
Se toman medidas para reaccionar ante las necesidades de los estudiantes, por ejemplo, traduciendo la información al inglés. Pero, con frecuencia, los mismos estudiantes no distinguen qué tipo de información es relevante para ellos.

Recibimos mucha información que no hemos solicitado. Pero si no sabemos que existe tal o cual información, tampoco sabemos qué pedir. (S10)

La red multicultural, en parte, logra cubrir este vacío de información:

La vida en la residencia universitaria es, en este caso, una ventaja. Puedes preguntar qué novedades hay. (S10)

Aunque los programas impartidos en inglés ya llevan más de un año de funcionamiento, este tipo de problemas continúa ... parte de la información en la página web está solo en lituano. Algunos reglamentos no están traducidos al inglés ... Esto es un gran inconveniente ... El personal administrativo está trabajando en ello. (A4)

Los representantes administrativos de la universidad constatan que, ante este problema, a los estudiantes siempre se les ayuda y se emplean los medios suficientes para que la información requerida esté disponible en inglés. Dicha labor consume tiempo y, a menudo, no se da abasto con la cantidad de información solicitada. Peor aún, parte de la información ya disponible en inglés va perdiendo actualidad:

A veces encuentras información en inglés, pero ya está desfasada, no ha sido actualizada. (A1)

Los obstáculos comunicacionales suelen derivarse también de problemas en los procesos de digitalización. Los mensajes electrónicos centralizados suponen un problema para los estudiantes que se sienten incómodos al recibir información en un idioma incomprensible:

Imagínese, recibe correos electrónicos en lituano y no los puede leer. Y a veces contienen información importante. Es raro, porque está dirigida a estudiantes extranjeros. (S9)

Obvio que se puede intentar traducir con el Google Translator, pero la traducción nunca será $100 \%$ correcta. (S6) 
Los estudiantes extranjeros desconocen las sutilezas en la gestión de información e interpretan estas situaciones como una falta de atención y cortesía. La sensación de marginalización y falta de atención se agudiza por la carencia de información relevante:

Te sientes como que no les importas. Tenemos que ir a rogarles que nos den información muy necesaria. (S9)

Con frecuencia esta información es obvia para los locales, pues la van recabando a lo largo de años de escolarización en su propio ambiente cultural. Al parecer, las novedades metodológicas en el aula no suponen un serio desafío para los extranjeros, pero sí la falta de información detallada sobre cómo realizar las tareas asignadas:

¿Cómo escribir un ensayo? ¿Qué estructura seguir? ¿Qué extensión? ... Este tipo de indicaciones son cosa sabida para los estudiantes locales, pero los extranjeros precisan más detalles. No hay información disponible sobre los requisitos para la tesina final de maestría. (S1) Y si existe, está en lituano. (S6)

Cabe señalar que los estudiantes extranjeros, al llegar a un nuevo país, tienen que adaptarse a la cultura local, hacerse de las costumbres y matices de la vida ordinaria de los lugareños y todo esto no es tarea fácil, pues el proceso de adaptación comporta grandes esfuerzos y mucha energía. Por eso, una parte inseparable de este proceso es el período de rechazo caracterizado por un aumento de la sensibilidad, inseguridad y necesidad de apoyo social. Contar con información requerida y detallada ayuda a reducir la incertidumbre y a que los estudiantes se sientan más seguros. Por otro lado, ante fracasos en lo más ordinario y la insatisfacción de necesidades básicas, se suele tener una visión más crítica y negativa de la situación.

La necesidad de información detallada es mayor cuando se desconocen los trámites burocráticos, a lo que se suma la falta de información en un idioma comprensible y la divergencia en experiencia sociocultural previa con respecto a la cultura local:

Sobre la tarjeta de estudiante internacional. ¿Quién es el encargado? Pienso que es responsabilidad de la universidad, pero aquí es distinto. 
Se encargan a otras organizaciones. (S9)

Nadie se toma la molestia de ayudarte, nadie tiene prisa por responder a tus preguntas y eso te hace sentir fatal ... He intentado varias instancias: me dirigí a la coordinadora de la Facultad, a la asociación de estudiantes, luego a la organización estudiantil en el centro de la ciudad. Me llevó más de un mes conseguir que me dieran la tarjeta de estudiante. (S3)

Los problemas surgidos por la falta de información en un idioma comprensible son causa de disgustos.

A veces te sientes indefenso y te desanimas cuando vas por tercera vez y... otra vez la puerta cerrada! (S4)

Problemas nimios que podrían resolverse con agilidad y facilidad llegan a ser irritantes. Sin embargo, para quien viene con una experiencia sociocultural distinta a la local, la información detallada es un recurso esencial y su carencia se torna exasperante. Lo mismo se aplica a los formularios, pues al rellenarlos surgen dudas y puede haber ciertos elementos de sensibilidad cultural.

Resulta que cuando rellenas un formulario hay distintos criterios para los locales y los extranjeros. Por ejemplo, si Ud. es lituano puede rellenar el número de identificación personal, pero si eres extranjero, ¿qué pones? Fui 3 o 4 veces a preguntar a la coordinadora ... la cuestión es que no hay instrucciones claras para rellenar ciertos datos si eres extranjero. (S5)

Este tipo de problemas y otros similares ocurren a menudo y los estudiantes no saben a quién dirigirse para conseguir la información requerida. Las fuentes principales de información para los alumnos extranjeros siguen siendo los gestores de estudios, a quienes se dirigen también con cuestiones no relacionadas con lo académico, ya que son las personas que tienen más cerca y que tal vez puedan responderles:

Hay muchas cuestiones personales. (A1)

Los estudiantes tienden a dirigirse con todo tipo de preguntas a personas con quienes se sienten seguros y acogidos, independientemente de la competencia y capacidad para resolverlo. (A1) 
Los gestores, a su vez, tratan de brindarles información, incluso cuando vienen con asuntos que no les corresponde a ellos tramitar (alojamiento, cuestiones de migración, etc.) pues si obraran de otro modo sólo contribuirían a acrecentar el estrés de los estudiantes ante la falta de información o el no saber dónde buscarla:

Y como viene no uno sino varios estudiantes con las mismas preguntas, ya en parte sabemos qué responderles. (A4)

Les digo lo que tienen que hacer y si no sé, llamo a algún colega que pueda saberlo para ahorrar al estudiante el disgusto o inconveniente de tener que acudir a otra oficina. (A5)

O los acompaño al otro despacho. (A1)

La comunicación asidua y el contacto personal con estudiantes extranjeros son importantes también para el personal administrativo, no sólo para los profesores. El personal administrativo entrevistado añadió que (aun cuando la información requerida está en inglés) los estudiantes extranjeros suelen dirigirse a ellos para pedir ayuda:

Preparamos hojas informativas con toda la información necesaria, basándonos en la experiencia del año anterior, con la idea de facilitarles las cosas a los de este año ... pero aun así vienen con las mismas preguntas. (A5)

El contacto humano y la explicación presencial son irremplazables, aunque la información requerida sea accesible:

A veces necesitan ayuda, no por la información en sí, sino porque no saben manejarse en el sistema informático. (A1)

A los estudiantes extranjeros se les asignan mentores voluntarios, también estudiantes, para que les asistan en problemas de la vida ordinaria. No obstante, la mayoría de los problemas están relacionados a cuestiones académicas concretas y específicas de cada Facultad o programa de estudios, por lo cual los mentores no siempre pueden ayudar:

Ellos no pueden saberlo todo. Le pregunté cómo utilizar el horario electrónico y ella dijo: "No sé”. (S9) 
Mi mentora es estudiante de medicina y no tiene nada que ver con mis estudios, puede ayudarme con cosas generales, pero tengo miles de preguntas específicas. (S2)

Y los mismos mentores no siempre son tan responsables. Tengo mentores, probablemente, pero aún ni nos hemos encontrado. (S10)

La información relativa a los estudios en la universidad se prepara con antelación y está dirigida a todos los estudiantes sin distinción. Las clases introductorias son muy informativas, pero durante el año lectivo van surgiendo una infinidad de situaciones en las que los estudiantes extranjeros no saben cómo comportarse ni dónde encontrar información en un idioma comprensible.

\section{Discusión y conclusiones}

Los resultados obtenidos permiten afirmar que las tendencias de globalización en el ámbito de la educación superior deberían ser acompañadas por una sólida preparación institucional para acoger el creciente número de estudiantes internacionales, empezando por garantizar (o al menos facilitar) la formación en competencias interculturales y lingüísticas del personal académico y administrativo.

Esto conlleva una seria revisión de la estrategia institucional para implementar los necesarios reajustes en los procesos administrativos que luego impactan, de manera directa, en el proceso educativo. Nos encontramos ante una transformación global del sistema educativo a la que se ha de prestar la debida atención para gestionar los cambios organizacionales que las IES ya están experimentando y que (con frecuencia) ocurren con más rapidez de lo que el gobierno institucional es capaz de dirigir a buen puerto.

En términos generales, se puede afirmar que los retos de los estudiantes que realizan estudios en inglés en universidades no angloparlantes son (en una significativa proporción) de índole comunicacional y no académica (métodos de enseñanza-aprendizaje apropiados o no). Nuevos o distintos métodos de enseñanza no suponen un desafío, puesto que los estudiantes están concientizados y esperan este tipo de novedades. En cambio, sí cobra relieve el modo de presentar las tareas que estos métodos proponen y la necesidad de que vayan acompañados de orientaciones claras y detalladas. 
Los ambientes académicos angloparlantes cuentan con una mayor proporción de estudiantes locales y pocos de otros países. En cambio, los estudiantes de programas impartidos en inglés en la Universidad de Vilnius se encuentran con una alta densidad de diversidad cultural dentro de su grupo académico, lo cual constituye un obstáculo para una comunicación efectiva. Esto acentúa la necesidad de conocimientos y competencia en comunicación intercultural. En un grupo de alta densidad multicultural, el profesor asume el rol de consultante-facilitador de comunicación intercultural. De ahí su necesidad imperiosa de conocimientos especializados y alta competencia intercultural.

Aunque la competencia lingüística en inglés (como idioma común de trabajo) sea importante, los entrevistados no lo consideran un elemento decisivo ni frustrante, como podría serlo en las universidades de países angloparlantes. Los profesores y el personal administrativo (quienes desempeñan sus tareas en un idioma distinto de su lengua materna) entienden la situación de los estudiantes extranjeros y se muestran dispuestos a brindarles ayuda y apoyo. Destaca como un reto específico la falta de dominio del idioma local, pues agudiza la exclusión comunicativa de los extranjeros. Aun sabiendo y pudiendo comunicarse en inglés con los extranjeros, las personas del país dan preferencia a su propio idioma. Así, la comunicación en inglés es muy fragmentaria y la interacción muy escasa.

La exclusión social y comunicativa de estudiantes locales y extranjeros se ve incrementada por la falta de motivación y atención hacia la creación de vínculos en la red bicultural y la transferencia de la necesidad de información y socialización hacia las redes monocultural y multicultural. Esto se da por analogía en aquellos que estudian en inglés en ambientes angloparlantes.

Los estudios impartidos en inglés presuponen la traducción al mismo de abundantes recursos informativos, lo cual implica un gran desafío para una universidad cuyos programas de estudios se imparten casi exclusivamente en lengua local, a excepción de su reducida oferta académica en inglés. De esta forma, la limitada disponibilidad de información en inglés acentúa la exclusión informativa, agudizada por la total desproporción entre la baja demanda de información de los estudiantes locales, en comparación la apremiante necesidad de comunicación más asidua e información exhaustiva que acusan los estudiantes extranjeros. 


\section{Fuentes consultadas}

Al-Shariden, K., \& Goe W. (1998). Ethnic communities within the university: An examination of factors influencing the personal adjustment of international students. Research in Higher Education, 39, 699-725.

Altbach, P. (2004). Higher Education Crosses Borders: Can the United States Remain the Top Destination for Foreign Students? The Magazine of Higher Learning, 36(2), 18-25.

Andrade, M. (2006). International students in English-speaking universities. Journal of Research in International Education, 5(2), 131-154.

Butcher, A., \& McGrath, T. (2004). International students in New Zealand: needs and responses. International Education Journal, 5(4), 540-551.

Centro de Análisis y Seguimiento de la Educación Superior e Investigación de Lituania - Mokslo ir studijų stebėsenos ir analizès centras (MOSTA, 2016). Lietuvos švietimas skaičiais. https://mosta.lt/lt/tyrimai/ ataskaitos\#2016-m

Chan, S. (1999). The Chinese learner - a question of style. Education and Training, 41, 294-304.

Chen, S., \& Absalom, D. (1996). Cultural awareness and language teaching methodology. En Issues in language education: Selected papers from ILEC'95 (pp. 171-190). Hong Kong Institute of Education.

Cowley, P., \& Hyams-Ssekasi, D. (2018). Motivation, Induction, and Challenge: Examining the Initial Phase of International Students' Educational Sojourn. Journal of International Students, 8(1), 109-130.

De Vita, G. (2001). Learning styles, culture and inclusive instruction in the multicultural classroom: a business and management perspective. Innovations in Education and Teaching International, 38(2), 165-174.

Departamento de Estadística de Lituania (2019). Lithuanian Statistical Agency. Official statistics portal. https://osp.stat.gov.lt 
Flora Hung, C. (2004). Cultural influence on relationship cultivation strategies: Multinational companies in China. Journal of Communication Management, 8(3), 264-281. https://doi. org/10.1108/13632540410807682

Furnham, A. (2004). Foreign students: Education and culture shock. The Psychologist, 17(1), 16-19.

Gilleard, J. (1998). Managing the cultural divide: The case of classroom assessment. Industrial and Commercial Training, 30(3), 90-95.

Graddol, D. (2006). English Next. Why global English may mean the end of English as a Foreign Language. British Council.

Halic, O., Greenberg, K., \& Paulus, T. (2009). Language and academic identity: A study of the experiences of non-native english speaking international students. International Education, 38(2), 73-93.

Hechanova-Alampay, R., Beehr, T., Christiansen, N., \& Van Horn, R. (2002). Adjustment and strain among domestic and international student sojourners: A longitudinal study. School Psychology International, 23(4), 458-474. https://doi.org/10.1177/0143034302234007

Kleiber, P. (2004). Focus groups: More than a method of inquiry. En K. De Marrais \& S. Lapan (Eds.), Foundations for research. Methods of inquiry in education and the social sciences. Lawrence Erlbaum Associates.

Ladd, P., \& Ruby R. (1999). Learning style and adjustment issues of international students. Journal of Education for Business, 74(6), 363-367. https://www.tandfonline.com/doi/ abs/10.1080/08832329909601712

Li, G., Chen, W., \& Duanmu, J. (2009). Determinants of international students' academic performance: A comparison between chinese and other international students. Journal of Studies in International Education, 14(4), 389-405. https://journals.sagepub.com/doi/ abs/10.1177/1028315309331490 
Myles, J., \& Cheng, L. (2003). The social and cultural life of non-native english speaking international graduate students at Canadian University. Journal of English for Academic Purposes, 2, 247-263.

Morgan, D. (1997). Focus groups as qualitative research. Sage Publications.

Pun, A. (1990). Managing the cultural differences in learning. Journal of Management Development, 9(5), 35-40.

Rajapaksa, S., \& Dundes, L. (2002). It's a long way home: International student adjustment to living in the United States. Journal of College Student Retention: Research, Theory and Practice, 4(1), 15-28. https:// journals.sagepub.com/doi/abs/10.2190/5HCY-U2Q9-KVGL-8M3K

Robertson, M., Line, M., Jones, S., \& Thomas, S. (2000). International students, learning environments and perceptions: A case study using the Delphi technique. Higher Education Research \& Development, 19(1), 89-101. https://www.tandfonline.com/doi/abs/10.1080/07294360050020499

Robotham, D. (1995). Self-directed learning: The ultimate learning style? Journal of European Industrial Training, 19(7), 3-7.

Sawir, E. (2005). Language difficulties of international students in Australia: The effects of prior learning experience. International Education Journal, 6(5), 567-580.

Selvadurai, R. (1992). Problems faced by international students in American colleges and universities. Community Review, 12(1-2), 27-32. https:// eric.ed.gov/?id=EJ469274

Trice, A. (2003). Faculty perceptions of graduate international students: Benefits and challenges. Journal of Studies in International Education, 7(4), 379-403. https://journals.sagepub.com/doi/ abs/10.1177/1028315303257120

Zhou, Y., Jindal-Snape, D., Topping, K., \& Todman, J. (2008). Theoretical models of Culture Chock and Adaptation in International Students in Higher Education. Studies in Higher Education, 33(1), 63-75. 